\title{
Excited state molecular dynamics simulations of nonlinear push-pull chromophores
}

\author{
Andrew M. Moran ${ }^{b, 1}$, Anne Myers Kelley ${ }^{\text {b }}$, Sergei Tretiak ${ }^{\mathrm{a}, *}$ \\ a Theoretical Division, Center for Nonlinear Studies, Los Alamos National Laboratory, Los Alamos, NM 87545, USA \\ ${ }^{\mathrm{b}}$ Department of Chemistry, Kansas State University, Manhattan, KS 66506-3701, USA
}

Received 2 May 2002; in final form 26 September 2002

\begin{abstract}
Julolidinemalononitrile, $p$-nitroaniline, and julolidinyl- $n-N, N^{\prime}$-diethylthiobarbituric acid are studied with ground and excited state molecular dynamics simulations in conjunction with the collective electronic oscillator formalism and Onsager's cavity model. Ground and excited state geometries are calculated in the gas phase and four solvents. The results are interpreted in the context of a two-state valence bond model for charge-transfer transitions of conjugated organic molecules, and are compared to recent resonant Raman experimental results. The calculated geometries are qualitatively consistent with both the two-state model and experiment. In addition, calculated transition density matrices are presented to visualize the changes in charge distribution accompanying photoexcitation.
\end{abstract}

(c) 2002 Elsevier Science B.V. All rights reserved.

\section{Introduction}

'Push-pull' organic molecules are characterized by the $\pi$-conjugated linkage of an electron donating and an electron accepting group. Because many push-pull chromophores exhibit large nonlinear optical responses, they are potentially useful for photonic and optoelectronic applications $[1,2]$. In addition to being easier to process than the inorganic crystals currently utilized, these molecules offer the advantage of property modulation through structural modification.

\footnotetext{
${ }^{*}$ Corresponding author. Fax: +1-505-665-4063.

E-mail address: serg@cnls.lanl.gov (S. Tretiak).

${ }^{1}$ Current address: Department of Chemistry, University of Rochester, Rochester, NY 14627-0216, USA.
}

The ground and lowest energy excited electronic states of push-pull molecules are often described in terms of molecular resonance forms. Neutral and zwitterionic basis states are represented by corresponding resonance forms, as shown in Fig. 1. The ground and charge-transfer (CT) excited states are defined as linear combinations of these two basis states [3-5]. For the systems considered in this work, the ground and CT states are predominantly composed of the neutral and zwitterionic states, respectively. Electron-phonon coupling is described by a bond length alternation (BLA) coordinate representing the linear dependence of conjugated bond order on basis state composition.

The optical properties of push-pull chromophores exhibit a strong dependence on solvation 


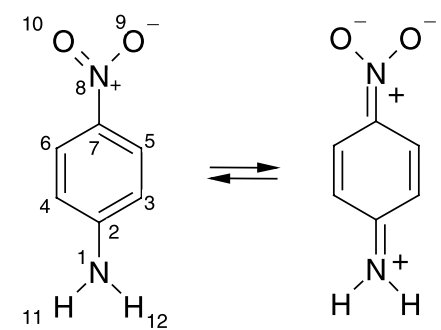

PNA

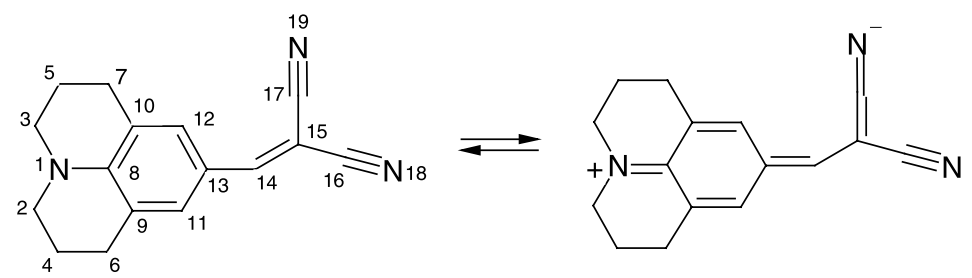

JM<smiles>CCN1C(=O)C(=Cc2cc3c4c(c2)CCCN4CCC3)C(=O)N(CC)C1=S</smiles><smiles>C#CC1CCC2=CC(=Cc3c([O-])n(CC)c(=S)n(CC)c3=O)C=C3CCC[NH+]=CC321</smiles>

JTB

Fig. 1. Resonance forms and atomic indices of PNA, JM, and JTB.

[3-9]. According to the two-state model, the zwitterionic character of the ground state should increase as solvent polarity increases, making the basis state compositions of both eigenstates more alike. Thus, as the environment becomes more polar, the energy difference between the two states decreases, and the geometries become more similar.

Previous theoretical work on related systems has been consistent with two-state trends. Cammi et al. [10] applied the polarizable continuum model to series of linear push-pull molecules, calculating ground state geometries both in vacuo and in water. They found the BLA coordinate changed coherently with both the strength of the electron donor/acceptor substituents and the inclusion of a solvent. Gao and Alhambra [9] obtained similar results for the some of the same molecules considered in [6] using a hybrid quantum mechanical and molecular mechanics methodology. Recent work on the betaine-30 dye molecule [11,12] has focused on electronic effects related to the torsional coordinate between its $\pi$ conjugated rings, and the importance of this contribution as a function of media polarity; the general conclusion was that the potential along the torsional coordinate has a much larger curvature in the isolated molecule than in solution. Farztdinov et al. [13] used semiempirical methods to explore the excited state potential energy surface of $p$-nitroaniline as a function of solvent. However, their focus was on torsional coordinates rather than the conjugated bond lengths.

Recently, the resonance Raman technique was applied to $p$-nitroaniline (PNA) [14], julolidinemalononitrile (JM) [15], and julolidinyl- $n-N$, $N^{\prime}$-diethylthiobarbituric acid (JTB) [16]. These analyses derive the dimensionless displacements between the ground and excited state potential surfaces for all the active vibrational modes by 
simultaneously fitting the absorption spectrum and Raman excitation profiles with an empirical model defined by a common set of parameters [17]. Qualitative agreement between experimental results and the two-state model was found. For nonstrongly hydrogen-bonding solvents, transition energies and reorganization energies exhibit the expected solvent dependence: as solvent polarity increases, the absorption bands shift to lower energies and the internal reorganization energies decrease. However, the solvent sensitivity of the ground state is much smaller than that of the excited state, particularly in the case of PNA. Specifically, the solvent dependence of the ground state vibrational frequencies is minute compared with the vibrational reorganization energies accompanying electronic excitation.

The technique of resonance Raman intensity analysis does not allow direct determination of absolute geometry changes in the CT state relative to the ground state, since the intensities depend only on the magnitudes and not the signs of the dimensionless mode displacements [17]. Determination of respective signs requires the selection of the most probable sign combination of the dimensionless displacements among the $2^{N}$ possibilities ( $N$ is the number of Raman active normal modes) and is practical only for small molecules, and/or requires high quality data on a large set of isotopic derivatives. In this work, we use a novel excited state molecular dynamics (MD) approach to calculate both ground and excited electronic state equilibrium geometries of JM, PNA, and JTB. The simulations are performed by combining the Austin Model 1 (AM1) semiempirical Hamiltonian with the collective electronic oscillator (CEO) method. Solvent effects are incorporated using the Onsager formulation of the self consistent reaction field (SCRF). Computational results for excited state nuclear dynamics are analyzed in terms of electronic charge motion using transition densities, and are interpreted using the BLA picture.

Section 2 briefly describes the computational method. We discuss the computed excited state structures of PNA, JM, and JTB in Section 3.1, solvatochromic effects are presented in Section 3.2, the results of MD simulations for PNA and JM are discussed in Sections 3.3 and 3.4, respectively, and the results are considered in the context of the two-state valence bond model in IIIE. Our conclusions are finally summarized in Section 4.

\section{Computational methods}

We begin by outlining our computational strategy and then give some general details of the techniques used. Using the GAUSSIAN 98 package [18], the DFT (B3LYP/6-311G**) approach and AM1 semiempirical method were employed to perform a ground state geometry optimization and normal mode analysis on each molecule. These calculations provided reference ground state geometries and energies of the isolated molecules (in vacuo). Excited state transition energies of the molecules were then calculated in vacuo and in solution with the CEO procedure $[19,20]$ using the INDO/S [21] and AM1 [22] semiempirical Hamiltonians and the Onsager reaction field [19,23,24] (if solvent corrections were requested). The necessary Hamiltonian matrix elements were generated with the ZINDO (INDO/S) [21] and MOPAC-93 (AM1) [25] codes. In order to provide a high level $a b$ initio reference point, electronic transitions of the isolated molecules were also calculated at the TDDFT (B3LYP/6-311G**) level. For the smaller molecules, PNA and JM, we used an MD algorithm to calculate equilibrium ground and excited state geometries in vacuo and in solution; the excited state MD [26] utilized the CEO procedure $[19,20]$ and an AM1 Hamiltonian, whereas the ground state potential energy surface was defined solely in terms of the AM1 Hamiltonian. Finally, theoretical geometry changes and transition densities were analyzed in the context of experimental results and a two-state valence bond model.

The CEO approach has been described in detail elsewhere [19]. This method is based on the timedependent Hartree-Fock approximation for many-electron problems and solves an equation of motion for the reduced single electron density matrix [27]

$\rho_{m n}(t)=\left\langle\Psi(t)\left|c_{m}^{+} c_{n}\right| \Psi(t)\right\rangle$, 
where $\Psi(t)$ is the many-electron wavefunction (time-dependent single Slater determinant driven by an external field), $c_{m}^{+}\left(c_{n}\right)$ are creation (annihilation) operators, and the indices $m, n$ refer to known basis functions (e.g., atomic orbitals (AOs) in site representation). The computational bottleneck of this approach is the diagonalization of the two-particle Liouville operator $L$

$L \xi_{v}=\Omega_{v} \xi_{\mathrm{v}}$.

Here the eigenfrequencies $\Omega_{v}$ are electronic transition frequencies between the ground state $|g\rangle$ and an excited state $|v\rangle$. The transition density matrices, or electronic modes $\left(\xi_{v}\right)$, obtained from Eq. (2.2) can be represented as

$\left(\xi_{v}\right)_{m n}=\left\langle v\left|c_{m}^{+} c_{n}\right| g\right\rangle$,

and reflect the changes in an electronic density for a given transition $[19,20]$. The eigenvalue problem (Eq. (2.2)) could be solved very efficiently using fast Krylov space algorithms (e.g., Lanczos or Davidson). Even though these calculations take all occupied and virtual orbitals into account (full active space), the computation of an excited state manifold is not much more numerically demanding than computation of the Hartree-Fock ground-state wavefunction.

A two-dimensional real-space analysis of calculated transition densities $\left(\xi_{v}\right)$ is useful for tracking electronic density variation due to photoexcitation and interpreting optical properties. The diagonal elements $\left(\xi_{v}\right)_{n n}$ represent the net charge induced in the $n$th $\mathrm{AO}$ by the external field. The off-diagonal elements $\left(\xi_{v}\right)_{m n}$ with $m \neq n$ represent the joint probability amplitude of finding an electron and a hole located at the $m$ th and $n$th AOs, respectively. Thus, the electronic normal modes provide a real-space picture of electronic transitions by showing accompanying motions of optically induced charges and electronic coherences $[19,20]$.

Excited state MD simulations [26] solve the classical equation of motion for the nuclear degrees of freedom

$M_{\alpha} \frac{\partial^{2} q_{\alpha}}{\partial t^{2}}+\beta \frac{\partial \mathrm{q}_{\alpha}}{\partial t}=F_{\alpha}(\mathbf{q})=-\frac{\partial E(\mathbf{q})}{\partial \mathrm{q}_{\alpha}}$, along the trajectory on the excited state molecular potential surface using a numerical velocity Verlet finite difference algorithm [28]. Here $q_{\alpha}$ and $M_{\alpha}$ represent the coordinate and mass of one of the $3 K$-6 vibrational normal modes ( $K$ being the number of atoms) and $E(\mathbf{q})=E_{\mathrm{k}}(\mathbf{q})+E_{\mathrm{e}}(\mathbf{q})$ is the excited state energy, $E_{\mathrm{k}}$ being the nuclear kinetic energy. We calculate the potential energy as $E_{\mathrm{e}}=E_{\mathrm{g}}(\mathbf{q})+\Omega_{v}(\mathbf{q})$, where $E_{\mathrm{g}}$ is the reference ground state energy and $\Omega_{v}$ is an electronic transition energy. The forces $F_{\alpha}$ on the right-hand side of Eq. (2.4) are computed numerically (as opposed to the analytical derivative technique) with the quantum chemical CEO procedure combined with the AM1 semiempirical method. This allows us to treat both ground and excited states within the same Hamiltonian model.

The computations follow excited state molecular dynamics in the isolated molecule with vanishing damping $(\beta=0)$ or motion in a viscous liquid where the damping is nonzero $(\beta \neq 0)$. The latter leads to the excited state optimal geometry. The same procedure with $\Omega_{v}=0$ results in the ground state optimal geometry. We utilized the Davidson algorithm for solving the eigenproblem Eq. (2.2) which allows fast computation of excited state energies and efficient excited state MD along all nuclear degrees of freedom in fairly large molecular systems. The MD simulations were initiated from the AM1 ground state equilibrium geometry of the isolated molecules, and were allowed to run for a sufficient length of time for equilibration to occur. Simulations of JM ran for a length of $1 \mathrm{ps}$ in $1 \mathrm{fs}$ steps, whereas simulations for PNA required $1.5 \mathrm{ps}$ in $1 \mathrm{fs}$ steps.

Solvent effects were incorporated using Onsager's solvent cavity model $[19,23,24]$. This model assumes the solute to be placed inside a spherical cavity surrounded by a dielectric medium, which is characterized solely by its dielectric constant. The Fock operator $F_{m n}^{0}$ of the isolated molecule is then modified by adding interaction energy between the solute and solvent, resulting in [24]

$F_{m n}=F_{m n}^{0}-\frac{\varepsilon-1}{2 \varepsilon+1} \frac{\boldsymbol{\mu}_{\mathrm{g}} \cdot \boldsymbol{\mu}_{m n}}{a_{0}^{3}}$,

where $\varepsilon$ is the dielectric constant and $\boldsymbol{\mu}_{\mathrm{g}}$ is the ground state dipole moment (the expectation value 
of the dipole operator $\left.\boldsymbol{\mu}_{m n}\right)$. The dielectric constants of cyclohexane, dichloromethane, and acetonitrile are 2.0, 9.1, and 37.5, respectively [29]. The radii of the solute cavities were determined using the Gaussian 98 package [18] which calculates the molecular volume inside a density contour of 0.001 electrons $/$ bohr $^{3}$ using Monte Carlo integration and associates that with an effective sphere radius. The reaction field, based on the spherical cavity approach, gives an essential description of the solute-solvent interaction energy when specific interactions such as hydrogenbonding are not important.

Finally, to compare the computed excited state geometries with experimental results, the dimensionless normal mode displacements were converted into explicit geometry changes using the ground state normal modes from a DFT (B3LYP/ 6-311G**) calculation as [14]

$\delta_{i}=5.8065 \sum_{j=1}^{N} Q_{j i}\left(\varpi_{j} m_{j}\right)^{-1 / 2} \Delta_{j}$,

where $j$ is an index for the normal mode (the summation over $j$ is restricted to the $N$ Raman active normal modes), $i$ is a coordinate index, $Q_{j i}$ is a matrix containing normal mode coefficients (in internal coordinates), $\Delta_{j}$ is the empirically determined dimensionless displacement of mode $j, m_{j}$ is the reduced mass of mode $j$ in a.m.u., $\varpi_{j}$ is the vibrational frequency of mode $j$ in $\mathrm{cm}^{-1}$, and $N$ is the number of Raman active normal modes. Only the magnitudes, and not the signs, of $\Delta_{j}$ are obtained from resonance Raman intensity analysis, leaving $2^{N}$ possible excited state geometries. However, the number of geometries can be reduced by eliminating geometries that are not consistent with predetermined constraints.

\section{Results and Discussion}

\subsection{Excited states of the isolated molecules}

Table 1 contains excitation energies and oscillator strengths of the five lowest energy transitions of PNA, JM, and JTB. The first row labels the method used to calculate the excitation energy, and the second row describes the technique utilized to optimize the ground state geometry; these are vertical excitations. In all cases, the CT transition is assigned to the lowest energy strongly allowed transition, and transition density matrices confirm these choices. These calculations were done to compare CEO excitation energies to a presumably more accurate method; it is especially important to check the performance of the CEO (AM1) because this method is used in the excited state MD simulations. The high level TDDFT calculations are the most rigorous, whereas the CEO calculations done with the INDO/S Hamiltonian, which is parameterized specifically for spectroscopic purposes, are expected to be accurate as well.

The CT transition energies, oscillator strengths, and relative state ordering of PNA are highly dependent on the method used to calculate them. The CT transition is easily identified using the TDDFT and CEO (INDO/S) methods. However, the two lowest energy transitions calculated with the CEO (AM1) method have nearly identical oscillator strengths, making the assignment of the CT state ambiguous at this geometry. This is a potential problem for the excited state MD. However, the important question is whether or not this ambiguity persists at the CT state equilibrium geometry; we found it does not. Of the three chromophores, the experimental gas phase CT transition energy has been reported for PNA only; Farztdinov et al. [13] report an absorption maximum of $4.24 \mathrm{eV}$ at an unspecified temperature. We observe good agreement between experiment and calculated CT state energy for PNA using the TDDFT (accuracy $0.17 \mathrm{eV}$ ) and the CEO (INDO/ S) (accuracy $0.13 \mathrm{eV}$ ) methods. The CEO (AM1) approach underestimates the $\mathrm{CT}$ transition energy by $0.7 \mathrm{eV}$.

Better agreement among the methods was found for JM and JTB. Most importantly, identification of the CT transition is unambiguous for these molecules. In the case of JM, the CT state is the lowest energy state according to all three methods, whereas the CT state of JTB is calculated as the second lowest energy state. 
Table 1

Calculated excitation energies and oscillator strengths of PNA, JM, and JTB



${ }^{\mathrm{a}} \mathrm{CT}$ transition.

\subsection{Solvatochromism}

Table 2 presents transition energies and solvatochromic shifts (relative to cyclohexane) of each molecule calculated using the CEO (AM1) procedure, and an AM1 reference ground state. The calculated absorption and fluorescence energies of PNA and JM correspond to nuclear geometries that are in equilibrium with the solvent continuum, whereas the absorption energy of JTB is calculated at the B3LYP/6-311G** geometry in vacuo. In the cases of PNA and JM, solvation effects on the ground and excited state structures are modeled using the MD algorithm described in Section 2.

At the ground state equilibrium geometry of PNA, the CT transition is readily identifiable as the second lowest energy transition in cyclohexane and dichloromethane, whereas it is the absolute lowest in energy in acetonitrile; the oscillator strength of the CT transition is greater than 20 
Table 2

Solvent dependent transition energies and solvatochromic shifts $(\mathrm{eV})$

\begin{tabular}{|c|c|c|c|c|}
\hline Solvent ${ }^{\mathrm{a}}$ & Calculated absorption & Exp. $^{\mathrm{b}}$ absorption & Calculated fluorescence & Exp. fluorescence ${ }^{c}$ \\
\hline & \multicolumn{4}{|c|}{$\begin{array}{c}\text { Transition energy/eV } \\
\text { solvatochromic shift/eV }\end{array}$} \\
\hline \multicolumn{5}{|c|}{ PNA } \\
\hline Isolated molecule & 3.5196 & $4.24^{\mathrm{d}}$ & 3.3441 & \\
\hline \multirow[t]{2}{*}{ Cyclohexane } & 3.4215 & 3.8505 & 3.2779 & - \\
\hline & 0.0000 & 0.0000 & 0.0000 & \\
\hline \multirow[t]{2}{*}{ Dichloromethane } & 3.2488 & 3.4156 & 3.1192 & - \\
\hline & 0.1727 & 0.4349 & 0.1587 & \\
\hline \multirow[t]{2}{*}{ Acetonitrile } & 3.1726 & 2.817 & 2.5339 & - \\
\hline & 0.2489 & 0.598 & 0.7440 & \\
\hline \multicolumn{5}{|c|}{$\mathrm{JM}$} \\
\hline Isolated molecule & 2.9622 & & 2.6502 & \\
\hline \multirow[t]{2}{*}{ Cyclohexane } & 2.8362 & 2.8437 & 2.5675 & 2.6402 \\
\hline & 0.0000 & 0.0000 & 0.0000 & 0.0000 \\
\hline \multirow[t]{2}{*}{ Dichloromethane } & 2.5841 & 2.7071 & 2.4081 & 2.5300 \\
\hline & 0.2521 & 0.1366 & 0.1594 & 0.1101 \\
\hline \multirow{2}{*}{ Acetonitrile } & 2.4810 & 2.7190 & 2.3529 & 2.4747 \\
\hline & 0.3552 & 0.1247 & 0.2146 & 0.1655 \\
\hline \multicolumn{5}{|c|}{$\mathrm{JTB}^{\mathrm{e}}$} \\
\hline Isolated molecule & 2.8001 & & & \\
\hline \multirow[t]{2}{*}{ Cyclohexane } & 2.7351 & 2.5149 & - & - \\
\hline & 0.0000 & 0.0000 & & \\
\hline \multirow[t]{2}{*}{ Dichloromethane } & 2.6140 & 2.3752 & - & - \\
\hline & 0.1211 & 0.1397 & & \\
\hline \multirow[t]{2}{*}{ Acetonitrile } & 2.5676 & 2.3797 & - & - \\
\hline & 0.1675 & 0.1352 & & \\
\hline
\end{tabular}

${ }^{a}$ The cavity radii were $3.97,4.92$, and $5.55 \AA$ for PNA, JM, and JTB, respectively.

${ }^{\mathrm{b}}$ Absorption spectra for PNA, JM, and JTB are given in [14-16], respectively.

${ }^{\mathrm{c}}$ Ref. [30].

${ }^{\mathrm{d}}$ Ref. [13].

${ }^{\mathrm{e}}$ The calculated absorption energies of JTB correspond to the B3LYP/6-311G** geometry in the isolated molecule; the molecule is not equilibrated with the solvent continuum.

times that of any other of the five lowest energy transitions. The solvent dependence of the relative state ordering is consistent with the two-state idea relating the extent of energy gap reduction to solvent polarity. At the excited state equilibrium geometry, the CT state is the lowest in energy in all solvents. While the absolute transition energy is relatively well calculated in cyclohexane, the solvatochromic shifts are severely underestimated in dichloromethane and acetonitrile.

The CT state of JM is calculated as the lowest energy state in all solvents at both the ground and excited state geometries. In contrast to PNA, in JM the solvatochromic shifts are overestimated at the ground state equilibrium geometry. The pres- ence of aliphatic groups in JM may limit solvent accessibility and inhibit solvation effects on the experimental transition energies, although the calculated solvation energies are also reduced because of the larger cavity radius (Eq. (2.5)).

In JTB, the calculated CT state is the second lowest in energy in cyclohexane, whereas it is the lowest energy state in the two more polar solvents. As in PNA, the solvent dependence of the state ordering is consistent with the two-state picture. The calculated solvatochromic shifts of JTB are in better agreement with the experimental measurements than in either of the other two molecules. However, the shifts of JTB are calculated at an identical geometry in all solvents, whereas the 
shifts of PNA and JM are calculated at solvated equilibrium geometries, making a direct comparison between these calculations less meaningful. Currently our MD code follows the dynamics of only the lowest excited state. Even though excited state MD of JTB is computationally feasible, we were not able to calculate the CT state geometry of JTB in vacuo or in the less polar solvents where the CT state is second lowest in energy. In addition, empirical analysis of the experimental data via Eq. (2.6) is not feasible for such large molecule. Therefore, we limit our subsequent discussion to the MD results for PNA and JM.

\subsection{Ground and excited state potential energy surfaces of PNA}

The calculated ground state equilibrium values and excited state changes of selected internal coordinates of PNA are shown in Tables 3 and 4, respectively. With one exception, the conjugated bond lengths of PNA are consistent with the notion that the zwitterionic character of the ground state increases in polar media. The calculated bond length changes also suggest that the excited state possesses greater zwitterionic basis state character than the ground state. The exception to this trend

Table 3

Calculated equilibrium ground state values for selected internal coordinates of PNA

\begin{tabular}{|c|c|c|c|c|}
\hline Coordinate $^{\mathrm{a}}$ & Isolated molecule & Cyclohexane & Dichloromethane & Acetonitrile \\
\hline $\mathrm{R}(1,2)$ & 1.378 & 1.3641 & 1.3542 & 1.3501 \\
\hline $\mathrm{R}(2,3)$ & 1.421 & 1.4255 & 1.4319 & 1.4348 \\
\hline $\mathrm{R}(2,4)$ & 1.421 & 1.4255 & 1.4319 & 1.4348 \\
\hline $\mathrm{R}(3,5)$ & 1.384 & 1.381 & 1.3765 & 1.3745 \\
\hline $\mathrm{R}(4,6)$ & 1.384 & 1.381 & 1.3765 & 1.3745 \\
\hline $\mathrm{R}(7,8)$ & 1.478 & 1.471 & 1.4596 & 1.4542 \\
\hline $\mathrm{R}(8,9)$ & 1.204 & 1.206 & 1.2104 & 1.2124 \\
\hline $\mathrm{R}(8,10)$ & 1.204 & 1.206 & 1.210 & 1.212 \\
\hline $\mathrm{A}(10,8,9)$ & 121.75 & 120.92 & 119.60 & 119.067 \\
\hline $\mathrm{A}(11,1,12)$ & 117.00 & 119.20 & 118.44 & 118.10 \\
\hline $\mathrm{NH}_{2}$ wag & 15.20 & 0.30 & 0.05 & 0.03 \\
\hline $\mathrm{NO}_{2}$ twist & 0.57 & 0.00 & 0.00 & 0.00 \\
\hline
\end{tabular}

${ }^{\text {a }}$ Bond lengths are in $\AA$ and angles are in degrees.

Table 4

Geometry changes for selected internal coordinates of PNA

\begin{tabular}{|c|c|c|c|c|c|c|c|}
\hline \multirow[t]{2}{*}{ Coordinate $^{\mathrm{a}}$} & \multirow{2}{*}{$\begin{array}{l}\text { Isolated molecule } \\
\text { Calculated }\end{array}$} & \multicolumn{2}{|c|}{ Cyclohexane } & \multicolumn{2}{|c|}{ Dichloromethane } & \multicolumn{2}{|l|}{ Acetonitrile } \\
\hline & & Calculated & Expt. $^{\mathrm{b}}$ & Calculated & Expt. $^{\mathrm{b}}$ & Calculated & Expt. $^{b}$ \\
\hline $\mathrm{R}(1,2)$ & -0.012 & -0.005 & -0.006 & -0.005 & -0.010 & -0.019 & -0.003 \\
\hline $\mathrm{R}(2,3)$ & 0.017 & 0.016 & 0.002 & 0.018 & -0.004 & 0.022 & -0.005 \\
\hline $\mathrm{R}(2,4)$ & 0.032 & 0.027 & 0.002 & 0.018 & -0.004 & 0.022 & -0.005 \\
\hline $\mathrm{R}(3,5)$ & -0.007 & -0.007 & -0.029 & -0.009 & -0.028 & -0.010 & -0.021 \\
\hline $\mathrm{R}(4,6)$ & -0.019 & -0.016 & -0.028 & -0.009 & -0.028 & -0.010 & -0.021 \\
\hline $\mathrm{R}(7,8)$ & -0.020 & -0.022 & -0.054 & -0.022 & -0.052 & 0.020 & -0.041 \\
\hline $\mathrm{R}(8,9)$ & 0.006 & 0.008 & 0.040 & 0.012 & 0.031 & 0.020 & 0.026 \\
\hline $\mathrm{R}(8,10)$ & 0.006 & 0.008 & 0.040 & 0.012 & 0.031 & 0.020 & 0.026 \\
\hline $\mathrm{A}(10,8,9)$ & -0.33 & -0.36 & -6.06 & -0.41 & -5.95 & -1.55 & -4.85 \\
\hline $\mathrm{A}(11,1,12)$ & 0.88 & -0.28 & 2.43 & -0.55 & -1.35 & -1.64 & 1.34 \\
\hline $\mathrm{NH}_{2}$ wag & -3.61 & -0.21 & - & -0.03 & - & 0.98 & - \\
\hline $\mathrm{NO}_{2}$ twist & -0.28 & 0.05 & - & 0.22 & - & 24.05 & - - \\
\hline
\end{tabular}

${ }^{\text {a }}$ Bond lengths are in $\AA$ and angles are in degrees.

${ }^{\mathrm{b}}$ Ref. [14]. 
occurs for the $\mathrm{C}-\mathrm{NO}_{2}$ bond in acetonitrile; the BLA model predicts this bond length should decrease upon excitation, while an increase is calculated. An increase in the $\mathrm{C}-\mathrm{NO}_{2}$ bond length was also calculated by Farztdinov et al. [13] using the COSMO solvation model and the SAM1 semiempirical Hamiltonian. The possibility that this unexpected result was due to the presence of two local minima along the $\mathrm{C}-\mathrm{NO}_{2}$ bond coordinate was investigated by running the simulations from a variety of initial $\mathrm{C}-\mathrm{NO}_{2}$ bond lengths. However, the simulations all converged to the same excited state geometry. The experimental coordinate changes in Table 4 are derived in [14]. In general, the experimental changes are much greater than those of the MD, which is not surprising considering that the MD also underestimates the solvatochromic shifts.

An $\mathrm{NH}_{2}$ wag angle was defined as (180$D(11,1,2,12)) / 2$, where $D$ denotes the dihedral angle. The wag angle corresponds to the hybridization of the nitrogen atom; this angle should be $35^{\circ}\left(0^{\circ}\right)$ for a pure $\mathrm{sp}^{3}\left(\mathrm{sp}^{2}\right)$ hybridization of nitrogen; $\mathrm{sp}^{3}$ and $\mathrm{sp}^{2}$ characters are associated with the neutral and zwitterionic basis states, respectively. In agreement with two-state predictions, the amino wag angle decreases from the ground to excited state in vacuo, and decreases in increasingly polar media in the ground state. The resonance Raman experiments [14] were unable to detect this mode due to laser scattering in the spectral region below $500 \mathrm{~cm}^{-1}$.

A $\mathrm{NO}_{2}$ twist angle was defined as $[D(10,8$, $7,6)-180+D(10,8,7,9)]$. This angle describes the torsion angle of the $\mathrm{NO}_{2}$ group relative to the plane of the ring. While the $\mathrm{NO}_{2}$ group is essentially in the plane of the ring in all media in the ground state, a significant increase in the excited state twist angle is calculated in acetonitrile. Although this result contradicts the idea that the double bond character of the $\mathrm{C}-\mathrm{N}$ bond should increase in the excited state, hindering rotation, it consistent with theoretical modeling by Farztdinov et al. [13] which reported a global minimum at $90^{\circ}$
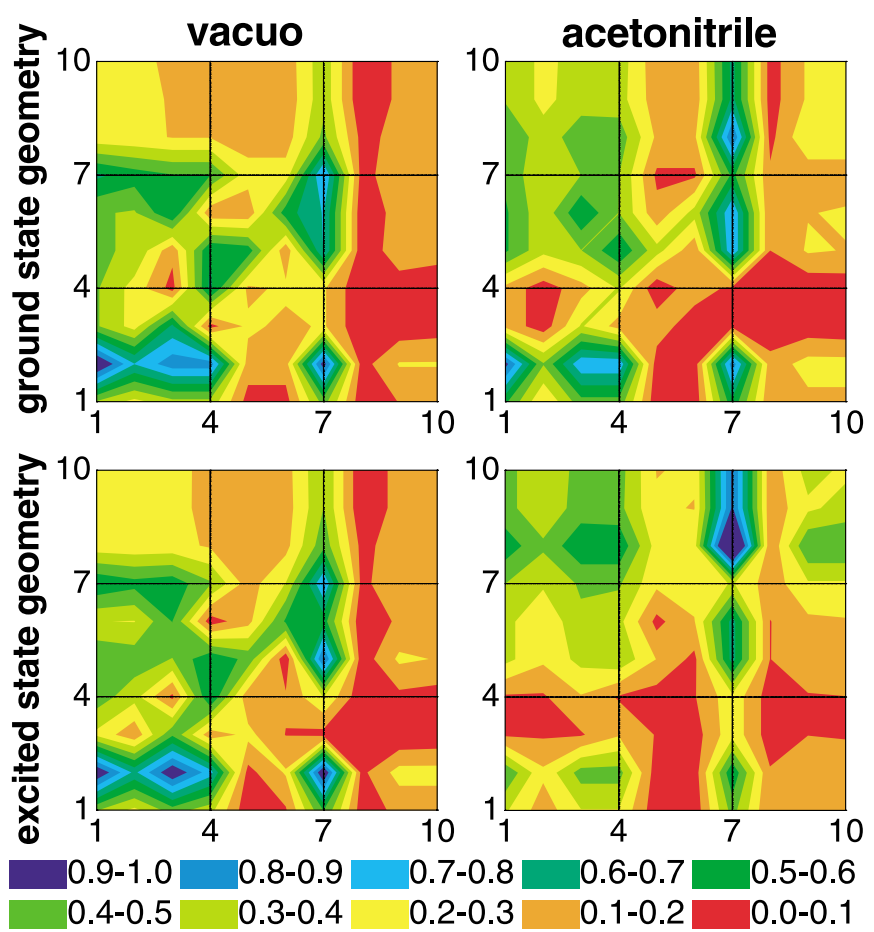

Fig. 2. Transition density matrices of PNA (absolute values of matrix elements, scaled to a maximum value of 1.0). See Fig. 1 for atomic indices. 
in water. Again, the resonance Raman experiments on PNA [14] could not detect such a low frequency mode $\left(<100 \mathrm{~cm}^{-1}\right)$.

To investigate how these geometrical changes are connected with the underlying photoinduced dynamics of electronic charges, we further use a two-dimensional real-space analysis of transition densities, representing the electronic transition between the ground state and an electronically excited state. Transition density matrices of PNA in vacuo and in acetonitrile, calculated at both the ground and excited state equilibrium geometries, are shown in Fig. 2. Each plot depicts probabilities of an electron moving from one molecular position (horizontal axis) to another (vertical axis) upon electronic excitation. For these CT transitions, the largest elements of the transition density matrix are expected to lie in the upper diagonal, representing electrons moving from a lower-numbered atom (closer to the donor group) to a highernumbered atom (closer to the acceptor group). Our primary interest is in the off-diagonal elements which are related to the probability amplitudes for a given CT event [19]. Since the signs of these matrix elements are not physically meaningful, we plot the absolute values of the transition density matrix elements using the contraction described in [19]. Therefore a diagonal element represents only the magnitude and not the sign of the charge induced on the atom by optical excitation. The color scale given in Figs. 2 and 3 associates a specific color to the range of magnitudes of matrix elements with the largest element set to 1.0 (blue color).

At the ground state geometry in vacuo, an offdiagonal maximum is located at $\rho_{21}$, which corresponds to an electron transfer from $\mathrm{N}_{1}$ to $\mathrm{C}_{2}$. The green area in the upper diagonal of the plot indicates unidirectional electron transfer from the donor to acceptor group. However, inspection of the $\rho_{m 7}(m=2,5,6)$ and $\rho_{2 n}(n=3,4,7)$ elements shows strong intramolecular charge transfer in the opposite direction, (in particular, from $\mathrm{C}_{2}$ to $\mathrm{C}_{7}$ ), which reflects rearranging the bond orders toward


Fig. 3. Same as Fig. 2, for JM. 
the zwitterionic state (Fig. 1). These CT patterns are slightly enhanced at the excited state geometry. At the ground state geometry, intramolecular charge transfer is stronger in acetonitrile than in the isolated molecule, particularly the elements $\rho_{m 7}$ $(n=8-10)$ which indicate electron transfer from $\mathrm{C}_{7}$ to $\mathrm{N}_{8}, \mathrm{O}_{9}$ and $\mathrm{O}_{10}$. In contrast, a significant reduction in the coherence magnitudes occurs at the excited state geometry in acetonitrile; this is especially evident in the $\rho_{m n}(n=1-4)$, whereas transfer from $\mathrm{C}_{7}$ to $\left(\mathrm{N}_{8}, \mathrm{O}_{9}\right.$, and $\left.\mathrm{O}_{10}\right)$ increases. This represents less CT from the donor group to anywhere else and stronger $\mathrm{CT}$ within the acceptor group. The increase in the $\mathrm{NO}_{2}$ twist angle is the coordinate change primarily responsible for this result. Increasing the $\mathrm{NO}_{2}$ twist angle interrupts the bond conjugation and reduces the CT character of the transition.

\subsection{Ground and excited state potential energy surfaces of $J M$}

The calculated ground state equilibrium values and excited state changes of selected internal coordinates of JM are shown in Tables 5 and 6, respectively. The solvent dependences of both the ground and excited state coordinate changes are qualitatively consistent with two-state predictions. Specifically, as solvent polarity increases, the ground state geometries display a more zwitterionlike structure and the magnitudes of the excited state coordinate changes decrease. The expected state dependence of the conjugated bond lengths is easily deduced by inspection of the resonance forms in Fig. 1.

In addition, an amino wag angle and single bond and double bond twist angles were defined for $\mathrm{JM}$ as $(180-D(2,1,8,3)) / 2, D(12,13,14,15)$, and $D(13,14,15,17)$, respectively. As in PNA, the amino wag angle is strongly correlated to the hybridization of the amino nitrogen; a decrease in this angle signifies the evolution from $\mathrm{sp}^{3}$ to $\mathrm{sp}^{2}$ hybridization, $\mathrm{sp}^{2}$ hybridization being associated with the zwitterionic basis state. At the ground state geometry, this angle changes coherently with solvent polarity in the expected two-state direction. The single bond and double bond twist angles also display the expected two-state solvent dependence at the ground state geometry: with increasing solvent polarity and increasing zwitterionic character, the $\mathrm{C}_{13}-\mathrm{C}_{14}$ bond order increases and the twist angle decreases, while the $\mathrm{C}_{14}-\mathrm{C}_{15}$ bond order decreases and the twist angle increases slightly. In addition, in all solvents the single bond twist angle decreases and the double bond twist angle increases in the excited state relative to the ground state, indicating a greater degree of zwitterionic character in the excited state.

Table 5

Calculated equilibrium ground state values for selected internal coordinates of JM

\begin{tabular}{lcccc}
\hline Coordinate $^{\mathrm{a}}$ & Isolated molecule & Cyclohexane & Dichloromethane & Acetonitrile \\
\hline $\mathrm{R}(1,8)$ & 1.391 & 1.383 & 1.368 & 1.363 \\
$\mathrm{R}(8,9)$ & 1.423 & 1.428 & 1.436 & 1.440 \\
$\mathrm{R}(8,10)$ & 1.424 & 1.426 & 1.433 & 1.437 \\
$\mathrm{R}(9,11)$ & 1.388 & 1.386 & 1.380 & 1.377 \\
$\mathrm{R}(10,12)$ & 1.391 & 1.389 & 1.384 & 1.381 \\
$\mathrm{R}(11,13)$ & 1.405 & 1.408 & 1.416 & 1.420 \\
$\mathrm{R}(12,13)$ & 1.401 & 1.402 & 1.407 & 1.410 \\
$\mathrm{R}(13,14)$ & 1.444 & 1.440 & 1.427 & 1.420 \\
$\mathrm{R}(14,15)$ & 1.355 & 1.357 & 1.364 & 1.370 \\
$\mathrm{R}(15,16)$ & 1.426 & 1.426 & 1.424 & 1.423 \\
$\mathrm{R}(15,17)$ & 1.422 & 1.420 & 1.418 & 1.416 \\
$\mathrm{R}(16,18)$ & 1.163 & 1.164 & 1.165 & 1.166 \\
$\mathrm{R}(17,19)$ & 1.164 & 1.164 & 1.165 & 1.166 \\
$\mathrm{C}_{2}-\mathrm{N}_{1}-\mathrm{C}_{3}$ wag & 13.18 & 9.57 & 4.11 & 2.05 \\
$\mathrm{C}_{13}-\mathrm{C}_{14}$ twist & 30.8 & 26.5 & 15.9 & 3.6 \\
$\mathrm{C}_{14}=\mathrm{C}_{15}$ twist & 2.5 & 2.8 & 3.5 & 3.6 \\
\hline
\end{tabular}

\footnotetext{
${ }^{\mathrm{a}}$ Bond lengths are in $\AA$ and angles are in degrees.
} 
Table 6

Geometry changes for selected internal coordinates of JM

\begin{tabular}{lcccccc}
\hline Coordinate $^{\mathrm{a}}$ & Isolated molecule & Cyclohexane & Methylene chloride & Acetonitrile & \multicolumn{2}{c}{$\begin{array}{c}\text { Experimental ranges } \\
\text { acetonitrile }\end{array}$} \\
\hline $\mathrm{R}(1,8)$ & & & & -0.007 & -0.011 & -0.010 \\
$\mathrm{R}(8,9)$ & -0.019 & -0.016 & -0.010 & 0.016 & 0.003 & 0.006 \\
$\mathrm{R}(8,10)$ & 0.023 & 0.022 & 0.019 & 0.014 & 0.0003 & 0.001 \\
$\mathrm{R}(9,11)$ & 0.019 & 0.018 & 0.016 & -0.008 & -0.004 & -0.002 \\
$\mathrm{R}(10,12)$ & -0.018 & -0.016 & -0.011 & -0.007 & -0.018 & -0.017 \\
$\mathrm{R}(11,13)$ & -0.016 & -0.015 & -0.011 & 0.017 & 0.002 & 0.005 \\
$\mathrm{R}(12,13)$ & 0.027 & 0.026 & 0.021 & 0.018 & 0.014 & 0.016 \\
$\mathrm{R}(13,14)$ & 0.026 & 0.025 & 0.021 & -0.015 & -0.014 & -0.006 \\
$\mathrm{R}(14,15)$ & -0.036 & -0.033 & -0.023 & 0.031 & 0.019 & 0.020 \\
$\mathrm{R}(15,16)$ & 0.032 & 0.033 & 0.033 & -0.010 & -0.0093 & -0.0088 \\
$\mathrm{R}(15,17)$ & -0.009 & -0.010 & -0.011 & -0.010 & -0.015 & -0.014 \\
$\mathrm{R}(16,18)$ & -0.012 & -0.012 & -0.012 & 0.005 & 0.005 & 0.006 \\
$\mathrm{R}(17,19)$ & 0.003 & 0.003 & 0.004 & 0.004 & 0.005 & 0.006 \\
$\mathrm{C}_{2}-\mathrm{N}_{1}-\mathrm{C}_{3}$ wag & -9.03 & 0.004 & 0.004 & -0.39 & $\mathrm{c}$ & $\mathrm{c}$ \\
$\mathrm{C}_{13}-\mathrm{C}_{14}$ twist & -14.6 & -6.48 & -1.15 & -1.6 & $\mathrm{c}$ \\
$\mathrm{C}_{14}=\mathrm{C}_{15}$ twist & 8.9 & -12.3 & -5.1 & 5.5 & $\mathrm{c}$ \\
\hline
\end{tabular}

${ }^{\text {a }}$ Bond lengths are in $\AA$ and angles are in degrees.

${ }^{\mathrm{b}}$ The two columns constitute the most negative (left) and positive (right) coordinate changes among the four possible geometries.

${ }^{\mathrm{c}}$ Mode was too low in frequency to be observed.

In order to evaluate the magnitudes of the calculated coordinate changes, the experimental normal mode displacements of JM [15] were projected onto a basis of B3LYP/6-311G** normal modes. The MD results were used to constrain the directions of the conjugated bond length changes and thus eliminate most of the 131,072 possible geometries (see Section 2). Using these constraints, and neglecting geometries with negligible changes in carbon-carbon bond lengths, all but four excited state geometries were eliminated in acetonitrile solvent. Table 6 contains the resulting ranges for selected coordinates. In general, agreement between the experimental projection and the MD simulations is much better for JM than for PNA. The relative performance of the MD may be linked to the differences in solvent accessibility between the two molecules. In the case of JM, the aliphatic substituents shield the donor and the acceptor is not as likely to interact strongly with the solvent. In other words, specific interactions are more important for PNA, making the solvent continuum model less appropriate for PNA than for JM.

Finally, we have examined the density matrices corresponding to the $\mathrm{CT}$ transition. The transition density matrices of JM at its ground and excited state equilibrium geometries both in vacuo and in acetonitrile are shown in Fig. 3. The appearance of the matrices is similar at both geometries, although the differences between media are most pronounced at the CT state geometry. The maximum at $\rho_{81}$ is a signature of the electron transfer from $\mathrm{N}_{1}$ to $\mathrm{C}_{8}$, analogous to the maximum at $\rho_{21}$ in PNA, and the green area in the upper diagonal of the plot reflects unidirectional electron transfer from the donor end of the molecule toward the acceptor end. There are some other large elements below the diagonal as well, indicative of charge transfer in the opposite direction from that expected for a donor-acceptor CT transition. These reflect charge redistribution between benzene ring and polyenic linker (e.g., $\rho_{8,13}$ and $\rho_{8,15}$ ). We also note that carbons $\mathrm{C}_{2}-\mathrm{C}_{7}$, which are not part of the conjugated $\pi$ system, do not participate in the optical transition. The conformationally important twist coordinates are reduced in acetonitrile which leads to an increase in the conjugation across the molecule, as opposed to what we observe in PNA. In particular, the increased planarity in solution promotes stronger charge transfer, at least in the vicinity of the polyenic linker. Prominent maxima are observed at both $\rho_{14,13}$ and 
$\rho_{14,15}$ in acetonitrile compared to that in vacuo at the ground state geometry, indicating a large charge redistribution between carbon 14 and both carbons 13 and 15 upon optical excitation. This redistribution reflects greater zwitterionic character in the excited state, i.e., increase of double (single) bond character for $\mathrm{C}_{13}-\mathrm{C}_{14} \quad\left(\mathrm{C}_{14}-\mathrm{C}_{15}\right)$ bonds. This trend is also observed at the excited state geometry in acetonitrile and is less pronounced in vacuo. Overall, the coupling of electronic states to the nuclear configuration is relatively complicated and no single coordinate of Tables 5 and 6 is sufficient to rationalize the changes in transition densities upon solvation.

\subsection{The two-state BLA picture of electron-phonon coupling}

Qualitative agreement with predictions of the two-state BLA model is found both computationally and experimentally, meaning that molecular structures become more zwitterionic in character as solvent polarity increases. However, the propriety of the BLA picture to chromophores with aromatic linker groups is unclear, at least in a quantitative sense. The BLA coordinate represents an evolution of bond order as a linear function of electronic state composition [3]. According to the formulation of Thompson et al. [5], the ground state of a push-pull chromophore is given as a linear combination of neutral $\left(\Psi_{\mathrm{N}}\right)$ and zwitterionic $\left(\Psi_{Z}\right)$ basis states

$\Psi_{\mathrm{g}}=f_{\mathrm{eq}}^{1 / 2} \Psi_{\mathrm{Z}}+\left(1-f_{\mathrm{eq}}\right) \Psi_{\mathrm{N}}$,

where $f_{\text {eq }}$ is the fraction of the zwitterionic basis state in the ground state at equilibrium. The BLA coordinate is a linear function of $f_{\text {eq }}$, and because the basis states are assumed to be orthogonal the ground state dipole $\left(\boldsymbol{\mu}_{\mathrm{g}}\right)$ is also a linear function of $f_{\text {eq }}[8,9,31]$, or $\boldsymbol{\mu}_{\mathrm{g}}=f_{\mathrm{eq}} \boldsymbol{\mu}_{\mathrm{Z}}+\left(1-f_{\mathrm{eq}}\right) \boldsymbol{\mu}_{\mathrm{N}}$.

Using the ground state dipoles obtained directly from the MD simulations, and estimates of the basis state dipoles $\left(\boldsymbol{\mu}_{\mathrm{Z}}\right.$ and $\left.\boldsymbol{\mu}_{\mathrm{N}}\right)$, assumed to be the same in all solvents, $f_{\text {eq }}$ 's of PNA were calculated (Table 7). Although our calculations predict a fairly strong dependence of the dipole moment upon solvent polarity, experimental measurements suggest the dipole moment is not so solvent-sensitive; the dipole moment has been measured at $6.3,6.29$, and $6.2 \mathrm{D}$ in the gas phase [32], benzene [33], and acetonitrile [7], respectively. The dipole moment of the zwitterionic basis state (30 D) state was calculated by assuming that full positive and negative charges are separated by a distance defined by the amino nitrogen and the midpoint between the two oxygen atoms, and the dipole of the neutral basis state $(2.36 \mathrm{D})$ was determined by summing the dipoles of aniline and nitrobenzene, calculated in the gas phase by AM1.

Plotting the conjugated bond lengths of PNA as a function of $f_{\text {eq }}$ yields decent linear fits. With the exception of the $\mathrm{N}_{1}-\mathrm{C}_{2}$ bond, linear fits of all plots have correlation coefficients greater than 0.99. While this result strongly coincides with the BLA formulation of electron-phonon coupling [3], the slopes of these plots are inconsistent with empirical observations. Experimental frequencies of nominal bond stretching modes of PNA change by less than $1 \%$ between these solvents, suggesting the bond lengths differ by no more than $0.3 \%$ [14]. We subsequently performed ground state normal mode analyses on PNA in solution using the B3LYP/6-311G** method and Onsager's model in several solvents [18]. Consistent with the results of our simulations, the calculated frequencies, which were linearly correlated to the basis state composition, were more solvent sensitive than those of the experiment. The calculations overestimated the experimental frequency differences between

Table 7

Electronic state compositions and ground state dipole moments (Debye)

\begin{tabular}{|c|c|c|c|c|c|c|c|c|}
\hline \multirow[t]{2}{*}{ Molecule } & \multicolumn{2}{|c|}{ In vacuo } & \multicolumn{2}{|c|}{ Cyclohexane } & \multicolumn{2}{|c|}{ Dichloromethane } & \multicolumn{2}{|c|}{ Acetonitrile } \\
\hline & $\boldsymbol{\mu}_{\mathrm{g}}$ & $f_{\text {eq }}$ & $\boldsymbol{\mu}_{\mathrm{g}}$ & $f_{\text {eq }}$ & $\boldsymbol{\mu}_{\mathrm{g}}$ & $f_{\text {eq }}$ & $\boldsymbol{\mu}_{\mathrm{g}}$ & $f_{\text {eq }}$ \\
\hline PNA & 7.295 & 0.18 & 8.350 & 0.22 & 10.060 & 0.28 & 10.680 & 0.33 \\
\hline JM & 7.223 & 0.13 & 8.441 & 0.16 & 10.450 & 0.22 & 11.220 & 0.24 \\
\hline
\end{tabular}


cyclohexane and acetonitrile by roughly a factor of two. It is not easy to rationalize the lack of solvent sensitivity the ground state geometry of PNA exhibits; both simple and sophisticated theories contradict experimental measurements. The failure of our model may be related to the use of a solvent continuum; explicit inclusion of solvent molecules should improve the description.

A similar analysis of the ground state composition was performed for JM. The dipole of the zwitterionic basis state (37.9 D) was calculated by assuming that full positive and negative charges were located on the amino nitrogen atom and the midpoint between the malononitrile nitrogen atoms. The dipole of the neutral basis state $(2.76 \mathrm{D})$ was defined by the sum of the AM1 dipoles of two molecules, a molecule formed by replacing the amino nitrogen of $\mathrm{JM}$ with $\mathrm{C}-\mathrm{H}$ and a molecule constructed by replacing the nitrile groups of $\mathrm{JM}$ with hydrogen atoms. The ground state dipoles and the corresponding $f_{\text {eq }}$ 's are presented in Table 7 .

The MD ground state simulations are also consistent with the two-state BLA picture. The linear dependence of the conjugated bond lengths of Table 5 on the basis state composition is strong; only plots of the nitrile bonds as a function of $f_{\text {eq }}$ had correlation coefficients less than 0.965 . The variability of the number of modes observed experimentally between the solvents negates the possibility of a straightforward comparison of projected excited state geometries with the simulated solvent dependence of the coordinates [15].

\section{Conclusions}

We have conducted molecular dynamics simulations of the ground and CT states of PNA and $\mathrm{JM}$ in vacuo and in solution using the semiempirical CEO (AM1) method for excited state calculations. The computational results are qualitatively consistent with the predictions of a simple two-state valence bond model and experimental results. Namely, the equilibrium geometry of the CT excited state has greater zwitterionic character compared to that of the ground state which results in corresponding changes of the bond-length al- ternation parameter. With one exception, all conjugated bond lengths change in the direction predicted by the two-state model, but the magnitudes of these coordinate changes are not in good agreement with empirical parameters derived from resonance Raman intensity analysis, particularly for PNA.

To study the effect of solvent polarity we used the Onsager reaction field, which is the simplest model for solvent corrections. Although calculated solvatochromic shifts are qualitatively correct, they are overestimated for PNA and underestimated for JM. In general, molecular structures become more (less) zwitterionic in character at the ground (CT excited) state equilibrium geometries as solvent polarity increases. Using the dipole moment to estimate basis state composition, good agreement with the simple twostate predictions is found with our relatively sophisticated model. However, both the simple two-state model and our computations drastically overestimate solvent effects on ground state nuclear geometries compared to those derived from experimental measurements. Our results suggest that the spherical cavity model is useful only for predicting qualitative trends involving solvents with significantly different polarities. More accurate COSMO [34] or polarizable continuum models [10], which correctly account for fast and slow components of dielectric response, and explicit solvent dynamic simulations are necessary to reproduce quantitative trends.

We find that conformational degrees of freedom such as twists are significantly affected by solvent. In PNA there is hardly any solvent dependence of the ground state conformation, while the excited state twists significantly in the highly polar solvent acetonitrile, contrary to two-state model predictions. In JM both ground and excited states become increasingly planar as the surrounding dielectric constant is increased. These conformational changes have a drastic effect on the electronic communication between donor and acceptor groups by promoting or reducing conjugation across the molecule. The impact of these conformations on intramolecular charge transfer can be well monitored by analysis of the corresponding transition densities. 
Even though all trends are reproduced qualitatively, the combination of the CEO approach with the AM1 semiempirical model does not produce quantitatively correct excited state energies, particularly for PNA. The INDO/S model works better for the PNA excited state energies, but this model does not work for the ground state. A good description of both ground and excited states within the same Hamiltonian model is essential for conducting excited state MD simulations. We further observe that the difference between the vertical excitation energies computed using INDO/ $\mathrm{S}$ and AM1 models is reduced for larger molecules (along the PNA, JM, JTB series) and is even smaller for larger molecular systems [35]. This trend can be explained by recalling that INDO/S has artificially decoupled $\pi$ and $\sigma$ terms in order to reproduce correctly the spectra of small molecules. For larger molecular systems beyond the strongly confined exciton regime [19] this decoupling becomes less important and INDO/S and AM1 results become similar. This makes the CEO (AM1) approach particularly promising for studying excited state potential surfaces in extended molecular systems.

\section{Acknowledgements}

This work was supported in part by ACF-PRF grant 33963-AC6 and NSF grant CHE-0109920 to A.M.K. We thank the Center for Nonlinear Studies and LDRD program at Los Alamos National Laboratory for financial support and computational facility usage.

\section{References}

[1] P.N. Prasad, D.J. Williams, Introduction to Nonlinear Optical Effects in Molecules and Polymers, Wiley, New York, 1991.

[2] J. Zyss (Ed.), Molecular Nonlinear Optics: Materials, Physics, and Devices, Academic Press, Boston, 1993.

[3] D. Lu, G. Chen, J.W. Perry, W.A. Goddard III, J. Am. Chem. Soc. 116 (1994) 10679.

[4] M. Barzoukas, C. Runser, A. Fort, M. Blanchard-Desce, Chem. Phys. Lett. 257 (1996) 531.

[5] W.H. Thompson, M. Blanchard-Desce, J.T. Hynes, J. Phys. Chem. A 102 (1998) 7712.
[6] S.R. Marder, J.W. Perry, G. Bourhill, C.B. Gorman, B.G. Tiemann, K. Mansour, Science 261 (1993) 186.

[7] M. Stähelin, D.M. Burland, J.E. Rice, Chem. Phys. Lett. 191 (1992) 245.

[8] M. Del Zoppo, C. Castiglioni, V. Gerola, P. Zuliani, G. Zerbi, J. Opt. Soc. Am. B 15 (1998) 308.

[9] J. Gao, C. Alhambra, J. Am. Chem. Soc. 119 (1997) 2962.

[10] R. Cammi, B. Mennucci, J. Tomasi, J. Am. Chem. Soc. 120 (1998) 8834.

[11] T. Ishida, P.J. Rossky, J. Phys. Chem. A 105 (2001) 558.

[12] J. Lobaugh, P.J. Rossky, J. Phys. Chem. A 104 (2000) 899.

[13] V.M. Farztdinov, R. Schanz, S.A. Kovalenko, N.P. Ernsting, J. Phys. Chem. A 104 (2000) 11486.

[14] A.M. Moran, A.M. Kelley, J. Chem. Phys. 115 (2001) 912.

[15] A.M. Moran, D.S. Egolf, M. Blanchard-Desce, A.M. Kelley, J. Chem. Phys. 116 (2002) 2542.

[16] A.M. Moran, C. Delbecque, A.M. Kelley, J. Phys. Chem. A 105 (2001) 10208.

[17] A.B. Myers, in: A.B. Myers, T.R. Rizzo (Eds.), Laser Techniques in Chemistry, Wiley, New York, 1995, p. 325.

[18] M.J. Frisch et al., Gaussian 98, Gaussian, Inc. Pittsburgh, PA, 1998.

[19] S. Tretiak, S. Mukamel, Chem. Rev. 102 (2002) 3171.

[20] S. Mukamel, S. Tretiak, T. Wagersreiter, V. Chernyak, Science 277 (1997) 781.

[21] M.C. Zerner, ZINDO, A Semiempirical Quantum Chemistry Program, Quantum Theory Project, University of Florida, Gainesville, FL, 1996.

[22] M.J.S. Dewar, E.G. Zoebisch, E.F. Healy, J.J.P. Stewart, J. Am. Chem. Soc. 107 (1985) 3902.

[23] L. Onsager, J. Am. Chem. Soc. 58 (1936) 1486.

[24] C.J.F. Böttcher, Theory of Electric Polarization, Elsevier, Amsterdam, 1973.

[25] J.J.P. Stewart, MOPAC, Schrodinger Inc. and Fujitsu Limited, Portland, 2000.

[26] S. Tretiak, A. Saxena, R.L. Martin, A.R. Bishop, Phys. Rev. Lett. 89 (2002) 97402.

[27] E.R. Davidson, Reduced Density Matrices in Quantum Chemistry, Academic, New York, 1976.

[28] M.P. Allen, D.J. Tildesley, Computer Simulation of Liquids, Clarendon Press, Oxford, 1987.

[29] CRC Handbook of Chemistry and Physics, Chemical Rubber Company, Boca Raton, FL, 1992.

[30] A.M. Moran, Ph.D. thesis, Kansas State University, Manhattan, KS, 2002

[31] F. Meyers, S.R. Marder, B.M. Pierce, J.L. Brédas, J. Am. Chem. Soc. 116 (1994) 10703.

[32] A.L. McClellan, Tables of Experimental Dipole Moments, W.H. Freeman, San Francisco, 1974.

[33] S. Millefiori, G. Favini, A. Millefiori, D. Grasso, Spectrochim. Acta A 33 (1977) 21.

[34] A. Klamt, G. Schüürmann, J. Chem. Soc. Perkin Trans. 2 (1993) 799.

[35] S. Tretiak, A. Saxena, R.L. Martin, A.R. Bishop, J. Chem. Phys. 115 (2001) 699. 